\title{
An Osteopenic Nonfracture Syndrome with Features of Mild Osteogenesis Imperfecta Associated with the Substitution of a Cysteine for Glycine at Triple Helix Position 43 in the Pro $\alpha$ 1(I) Chain of Type I Collagen
}

\author{
Jay R. Shapiro, ${ }^{*}$ Mary Louise Stover, ${ }^{\star}$ Virginia E. Burn, ${ }^{*}$ Monique B. McKinstry, ${ }^{*}$ \\ Allen L. Burshell, ${ }^{5}$ Stewart D. Chipman, ${ }^{*}$ and David W. Rowe ${ }^{*}$ \\ *Bone Metabolism Research Laboratory, Division of Geriatric Medicine \& Gerontology, Johns Hopkins University \\ School of Medicine, Baltimore, Maryland 21224; ${ }^{\ddagger}$ Department of Pediatrics, University of Connecticut Health Science Center. \\ Farmington, Connecticut 06032; ${ }^{\S}$ The Ochsner Clinic, New Orleans, Louisiana 70121
}

\begin{abstract}
Mutations affecting the pro $\alpha 1$ (I) or pro $\alpha 2(I)$ collagen genes have been identified in each of the major clinical types of osteogenesis imperfecta. This study reports the presence of a heritable connective tissue disorder in a family with an osteopenic syndrome which has features of mild osteogenesis imperfecta but was considered idiopathic osteoporosis in the proband. At age 38, while still premenopausal, she was found to have osteopenia, short stature, hypermobile joints, mild hyperelastic skin, mild scoliosis, and blue sclerae. There was no history of vertebral or appendicular fracture. Hip and vertebral bone mineral density measurements were consistent with marked fracture risk. Delayed reduction SDS-PAGE of pepsin-digested collagens from dermal fibroblast cultures demonstrated an anomalous band migrating between $\alpha 1(\mathrm{I})$ and $\alpha 1(\mathrm{III})$. This band merged with the normal $\alpha$-chains upon prereduction, indicating an unexpected cysteine residue. Cyanogen bromide peptide mapping suggested that the mutation was in the smaller $\mathrm{NH}_{2}$-terminal peptides. cDNA was reverse transcribed from mRNA and amplified by the polymerase chain reaction. $A$ basepair mismatch between proband and control $\alpha 1($ I) $\mathrm{cDNA}$ hybrids was detected by chemical cleavage with hydroxylamine:piperidine. The cysteine substitution was thus localized to $\alpha 1$ (I) exon 9 within the cyanogen bromide 4 peptide. Nucleotide sequence analysis localized a $G \rightarrow T$ point mutation in the first position of helical codon 43 , replacing the expected glycine ( $G G T)$ residue with a cysteine (TGT). The prevalence of similar $\mathrm{NH}_{2}$-terminal mutations in subjects with this phenotype which clinically overlaps idiopathic osteoporosis remains to be determined. (J. Clin. Invest. 1992. 89:567-573.) Key words: osteoporosis $\bullet$ bone $\bullet$ joint laxity $\bullet$ sclerae $\bullet$ heritable disorder
\end{abstract}

\section{Introduction}

Osteogenesis imperfecta $(\mathrm{OI})^{1}$ has been subdivided into four major clinical types of disease based on clinical severity, inheri-

Address correspondence to Dr. Jay R. Shapiro, Bone Metabolism Research Laboratory, Division of Geriatric Medicine and Gerontology, Johns Hopkins University Medical School, Asthma \& Allergy Center, 5A.64, 301 Bayview Boulevard, Baltimore, MD 21224.

Received for publication 30 April 1991 and in revised form 30 September 1991.

J. Clin. Invest.

(c) The American Society for Clinical Investigation, Inc.

0021-9738/92/02/0567/07 \$2.00

Volume 89, February 1992, 567-573 tance, and scleral color (1). However, it is recognized that these different phenotypes represent a continuum of disease severity. Toward the mild end of the spectrum it is frequently difficult to distinguish patients with mild OI type I from certain patients with idiopathic osteoporosis who present a family history of osteoporosis and fractures (2-4). These individuals may also have variable degrees of joint laxity and mild scoliosis. Blue sclerae are characteristic of OI type I, but this also occurs in other connective tissue disorders, as well as in otherwise normal individuals (5).

Most individuals with $\mathrm{OI}$ have mutations that affect the synthesis of type I collagen, the main structural protein of bone, tendon, and skin (6). Clinically, the overlap of findings in mild OI and idiopathic osteoporosis suggests that similar defects in type I collagen might be found in some osteopenic individuals whose mild skeletal disorder differs from that expected in subjects with OI.

The basic unit of the type I collagen triple helix is the triplet $(\mathrm{Gly}-\mathrm{X}-\mathrm{Y})_{\mathrm{n}}$, in which the repeat of the first position glycine is essential to the unique triple helical structure. Mutations involving the primary structure of the type I collagen gene may result in amino acid substitutions, insertions, deletions, mRNA splicing defects, and/or alterations in protein posttranslational modification (6). First position glycine substitutions have been recognized to account for the majority of $\mathrm{OI}$ cases reported (7). Point mutations in the first position of the glycine codon (GGN, where $\mathrm{N}$ can be any nucleotide) will change that residue to either cysteine $(G \rightarrow T)$, arginine $(G \rightarrow$ $C)$, or serine $(G \rightarrow A)$. On the other hand, a cysteine residue in the $\mathrm{X}$ or $\mathrm{Y}$ position could result from a single base substitution in the codons for glycine, arginine, phenylalanine, or serine. A characteristic disulfide-linked dimer, easily recognizable on SDS-PAGE, is formed when two pro $\alpha 1(\mathrm{I})$ chains containing a cysteine mutation associate with a normal pro $\alpha 2$ (I) chain. The impact of a cysteine substitution on skeletal matrix development will vary with the location of the mutation, i.e., either within or outside of the triple helical domain or toward the $\mathrm{COOH}$ or $\mathrm{NH}_{2}$ terminus (7).

This report describes a woman with an osteopenic syndrome consistent with mild OI type I, but in whom ascertainment was based on a diagnosis of idiopathic osteoporosis. She and her two affected children have never experienced fractures. The biochemical defect is a glycine 43 to cysteine mutation in one allele of $\alpha \mathrm{l}(\mathrm{I})$ collagen, located near the $\mathrm{NH}_{2}$ terminus of

1. Abbreviations used in this paper: $\mathrm{BMD}$, bone mineral density; $\mathrm{CB}$, cyanogen bromide; OI, osteogenesis imperfecta; PCR, polymerase chain reaction. 
the molecule in exon 9 (within cyanogen bromide [CB] peptide 4 ). We believe that patients presenting with this particular osteoporotic phenotype should be studied for type I collagen defects, particularly $\mathrm{NH}_{2}$-terminal mutations that would be predicted to have less severe consequences on collagen matrix development and skeletal formation.

\section{Methods}

Case report. The pedigree for this family is presented in Fig. 1. The proband (II-2, see arrow) was a 38-yr-old female whose clinical history includes joint laxity, dislocations of her shoulder, multiple ankle sprains, and a tear of her bladder and uterus during her last delivery. Significantly, there was no history of fractures. Menstrual history was normal. Physical examination revealed short stature $(151 \mathrm{~cm})$, blue sclerae, and joint laxity. There was no dentinogenesis imperfecta, scoliosis, or deformity of her limbs. The diagnosis of osteoporosis was based on osteopenia of the thoracic vertebrae initially seen on routine lateral chest $\mathrm{x}$ rays. Osteopenia was confirmed by dual photon densitometry of the lumbar vertebrae and hip. Mean vertebral bone mineral density (BMD) of L2-L4 was $0.792 \mathrm{~g} / \mathrm{cm}^{2}$ or $69 \%$ of age- and sexmatched normal controls. Mean femur (Wards's triangle) BMD was $0.650 \mathrm{~g} / \mathrm{cm}^{2}$ or $66 \%$ of age- and sex-matched normal controls. Normal control BMD values were those of the Oschner Clinic using a dual photon scanner (Lunar Co., Madison, WI) with a Gadolinium-153 source. These values are two standard deviations below that for ageand sex-matched normal controls and are consistent with marked fracture risk (8).

Two of her four children (Fig. 1, III-1 and III-4) share the phenotype of short stature (both were less than the fifth age adjusted percentile in height), joint laxity, dislocations, and blue sclerae. III-1 also had scoliosis. These two children had mean BMD in L2-L4 of 0.605 and 0.442 , respectively. These values are two standard deviations below that for young normal controls and are consistent with marked fracture risk (9). Neither of these children had experienced a fracture in spite of normal physical activity. The proband's other two children, husband, father,

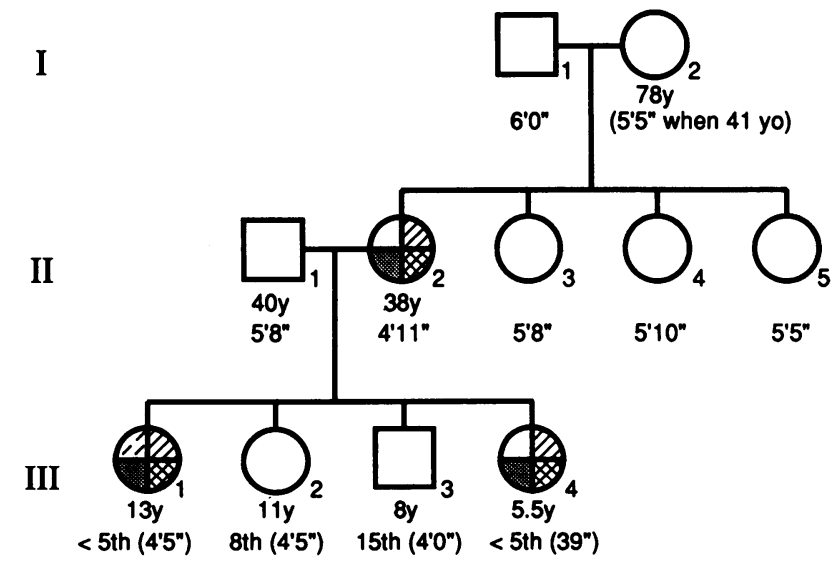

KEY:

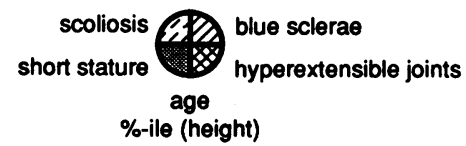

Figure 1. Pedigree for proband's family. The proband is II-2. Ages and heights are as indicated; for the III generation the height is preceded by age-matched percentile rank. and mother were normal with no history of fractures or skeletal deformity.

Fibroblast culture growth, radiolabeling, and harvesting. Dermal fibroblast cultures were isolated and maintained as previously described (10). Newly synthesized type I and III collagens were measured in fibroblast cultures in the third to fifth passages. Cultures were grown to visual confluence and radiolabeled with $0.5 \mu \mathrm{Ci}$ of $\mathrm{L}-\left[\mathrm{U}-{ }^{14} \mathrm{C}\right]$ proline/ $\mathrm{cm}^{2}$ of culture dish (Amersham Corp., Arlington Heights, IL) for $24 \mathrm{~h}$ in Delbecco's MEM containing 1\% dialyzed fetal bovine serum, 50 $\mathrm{U} / \mathrm{ml}$ penicillin, $50 \mu \mathrm{g} / \mathrm{ml}$ streptomycin, $150 \mu \mathrm{M}$ ascorbic acid, and $175 \mu \mathrm{M} \beta$-aminoproprionitrile. The cell layer and media were harvested and combined and proteolytic inhibitors were added to a final concentration of $5 \mathrm{mM}$ EDTA, $1 \mathrm{mM}$-hydroxymercuribenzoate, 5 $\mathrm{mM} n$-ethylmaleimide, and $100 \mu \mathrm{M}$ PMSF. The sample was then homogenized with a Broeck tissue grinder (Thomas Scientific, Swedesboro, $\mathrm{NJ}$ ) and precipitated with $30 \%$ ethanol (vol/vol) for electrophoretic analysis of the collagen $\alpha$-chains. Tissue culture supplies were obtained from Life Technologies, Inc. (Gaithersburg, MD) and other chemicals from Sigma Chemical Co. (St. Louis, MO) except as noted.

Type I collagen protein analysis. Ethanol-precipitated, $\left[{ }^{14} \mathrm{C}\right]$ proline-labeled samples were subjected to limited pepsin digestion $(10 \mu \mathrm{g} /$ $\mathrm{ml}$ pepsin for $6 \mathrm{~h}$ at $4^{\circ} \mathrm{C}$ ) followed by $2 \mathrm{M} \mathrm{NaCl}$ precipitation and $7.5 \%$ SDS-PAGE in either the unreduced condition, prereduced with DTT, or with delayed reduction (11). Two-dimensional 7.5\% SDS-PAGE was performed with delayed reduction in the first dimension, followed by DTT prereduction before the second dimension 7.5\% SDS-PAGE (12). Both dimensions were 7.5\% SDS-PAGE. Individual collagen $\alpha$ chains were peptide mapped by in situ $\mathrm{CB}$ digestion and 10-15\% gradient SDS-PAGE in the unreduced and reduced states (12). Autoradiographs of the radiolabeled collagens were analyzed by scanning densitometry (13). Type I collagen triple helix thermal stability was determined by the method of Bruckner and Prockop (14).

$m R N A$ isolation. Total fibroblast RNA from the proband and a control was isolated from confluent cell layers that had been treated for $24 \mathrm{~h}$ with ascorbate. The cell layer was then harvested in $1 \%$ SDS, 1 mM EDTA, $50 \mathrm{mM}$ Tris, $\mathrm{pH} 7.5$, buffer containing $100 \mu \mathrm{g} / \mathrm{ml}$ proteinase $\mathrm{K}$. The sample was incubated at $37^{\circ} \mathrm{C}$ for $60 \mathrm{~min}$ followed by phenol/chloroform extraction and ethanol precipitation of the nucleic acids (15). The nucleic acid pellet was solubilized in $6 \mathrm{M}$ guanidine$\mathrm{HCl}$ and the RNA separated from the DNA by selective precipitation with $0.5 \mathrm{vol}$ of ethanol. The RNA was sequentially washed in $4 \mathrm{M}$ guanidine/33\% ethanol, then $80 \%$ ethanol, dried in a vacuum (Speedvac SC100; Savant Instruments, Farmingdale, NY), redissolved in $\mathrm{H}_{2} \mathrm{O}$, and stored at $-70^{\circ} \mathrm{C}$.

$m R N A$ reverse transcription to $c D N A$. $\alpha \mathrm{l}(\mathrm{I})$ fibroblast $\mathrm{mRNA}$ was isolated from the proband and a control, and then reverse transcribed to cDNA using a 35-mer primer (3'-GG GAG TTC TCG GAC TCG GTC GTC TAG CTC TTG TAG-5') complementary to the $\mathrm{COOH}$ terminal propeptide (nucleotides 3,820-3,854). This fragment is located 46 nucleotides $3^{\prime}$ to the C-propeptide cleavage site. Approximately $5 \mu \mathrm{g}$ of total RNA was added to $2.5 \mu \mathrm{g}$ of oligonucleotide; the sample was heated to $65^{\circ} \mathrm{C}$ for $3 \mathrm{~min}$ and then cooled on ice. The volume was then adjusted to $50 \mu \mathrm{l}$ final volume with $50 \mathrm{mM}$ Tris, $\mathrm{pH}$ $8.3,75 \mathrm{mM} \mathrm{KCl}, 3 \mathrm{mM} \mathrm{MgCl}_{2}, 10 \mathrm{mM}$ DTT, and $500 \mu \mathrm{M}$ dNTPs. First strand cDNAs were synthesized by the addition of $450 \mathrm{U}$ of Maloney murine leukemia virus reverse transcriptase (Life Technologies, Inc.) followed by incubation for $60 \mathrm{~min}$ at $37^{\circ} \mathrm{C}(16)$.

Polymerase chain reaction $c D N A$ amplification. For polymerase chain reaction (PCR) amplification of the cDNA, $\sim 5 \mu$ l of the cDNA synthesis reaction was mixed with $100 \mathrm{pmol}$ of each of two oligonucleotides that flanked the nucleotide sequence of interest. The upstream oligonucleotide (5'-TCA TGA CAT CTA GAC TTT GCT CCC CAG CTG CTG TCT TAT G-3') was derived from exon 6, while the downstream oligonucleotide (5'-CGA GCA CCT TTA CTA CCA CGA TGA CCT AGG TCA CTG AT-3') was derived from exon 15 . These primers were designed to yield a cDNA of $538 \mathrm{bp}$ in length, spanning exons 6-15. The sample was diluted to $100 \mu 1$ with $16.6 \mathrm{mM}$ ammo- 
nium acetate, $67 \mathrm{mM}$ Tris- $\mathrm{HCl}, \mathrm{pH} 8.8,6.7 \mathrm{mM} \mathrm{MgCl}_{2}, 10 \mathrm{mM} \beta$ mercaptoethanol, $6.7 \mu \mathrm{M}$ EDTA, $33 \mu \mathrm{M}$ dNTPs, and $1.5 \mathrm{U}$ of Thermus aquaticus DNA polymerase (Perkin-Elmer Cetus, Emeryville, CA) was added and the reaction was run in a thermalcycler (17). After an initial denaturation at $94^{\circ} \mathrm{C}$ for $5 \mathrm{~min}, 30$ cycles were run with $1 \mathrm{~min}$ denaturation at $93^{\circ} \mathrm{C}, 1 \mathrm{~min}$ annealing at $62^{\circ} \mathrm{C}$, and $1 \mathrm{~min}$ polymerization at $72^{\circ} \mathrm{C}$, followed by final extension at $72^{\circ} \mathrm{C}$ for $7 \mathrm{~min}$.

Chemical cleavage of $c D N A$ heteroduplexes. The products of each PCR reaction were examined in a 5\% Tris/borate/EDTA/polyacrylamide minigel and the resulting exon 6-15 cDNA was electroeluted. The purified 6-15 exon cDNA fragment from wild-type cDNA was ${ }^{32} \mathrm{P}$ end-labeled using T4 DNA polymerase (18). Approximately $10 \mathrm{ng}\left(10^{6}\right.$ $\mathrm{cpm}$ ) of the wild-type cDNA probe was mixed with $120 \mathrm{ng}$ of the exon 6-15 cDNA fragment from the proband or an equal quantity of unlabeled, control exon 6-15 cDNA fragment. The final volume was $50 \mu \mathrm{l}$ of $300 \mathrm{mM} \mathrm{NaCl}, 3 \mathrm{mM}$ Tris, $\mathrm{pH} 7.7$, and $3.5 \mathrm{mM} \mathrm{MgCl}_{2}$. The sample was boiled for $5 \mathrm{~min}$ and allowed to anneal for $60 \mathrm{~min}$ at $42^{\circ} \mathrm{C}$ to form a cDNA heteroduplex. The sample was then precipitated with ethanol, redissolved in $18 \mu \mathrm{l} \mathrm{H}_{2} \mathrm{O}$, and divided into three 6- $\mu \mathrm{l}$ aliquots before the chemical cleavage procedure (19). $2.5 \mathrm{M}$ hydroxylamine was prepared by dissolving hydroxyl ammonium chloride (Analar grade BDH) in diethylamine. Each aliquot of the sample had $20 \mu$ l of hydroxylamine added and was then incubated for 0,20 , and $60 \mathrm{~min}$. The reaction was stopped at each time point by addition of $200 \mu \mathrm{l}$ of $300 \mathrm{mM} \mathrm{Na}$ acetate, $100 \mathrm{mM}$ EDTA, and $25 \mu \mathrm{g} / \mathrm{ml}$ tRNA. The hydroxylamine modified cytosine nucleotides were cleaved by addition of $1 \mathrm{M}$ piperidine (Aldrich Chemical Co., Milwaukee, WI) at $90^{\circ} \mathrm{C}$ for $30 \mathrm{~min}$. After the nucleic acids were precipitated by ethanol they were examined on a $5 \%$ denaturing acrylamide gel and visualized by autoradiography (13).

Nucleotide sequencing. The PCR-derived fragment from exon 6-15 of cDNA from the proband was cloned by blunt end ligation into the SmaI site of a pBlueScript II KS(+) plasmid (Stratagene Inc., La Jolla, CA). The plasmid was transfected into $\mathrm{DH} 5 \alpha$ competent bacteria and colonies containing the insert were grown to harvest plasmid for DNA sequencing. Double stranded DNA sequencing was employed using a T7 Sequenase kit (United States Biochemical Corp., Cleveland, $\mathrm{OH}$ ).

\section{Results}

Protein analysis. 7.5\% SDS-PAGE of unreduced, pepsin-digested type I collagen $\alpha$-chains demonstrated a slowly migrat-

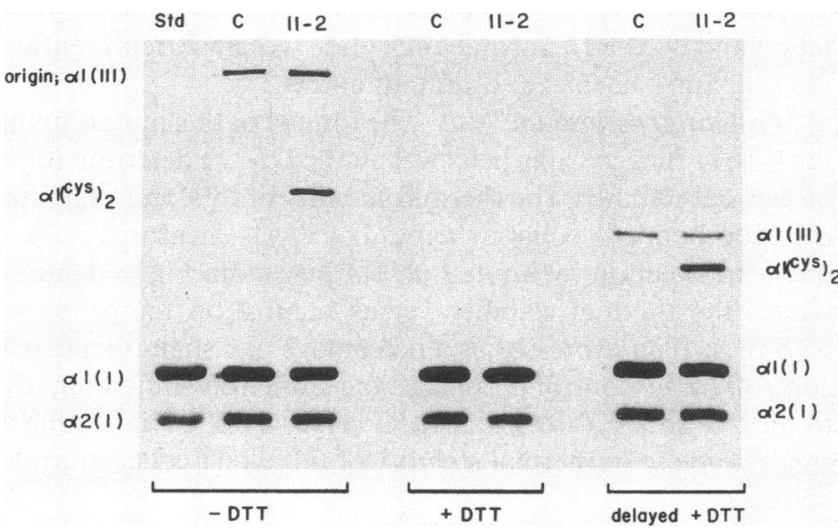

Figure 2. SDS-PAGE of collagen isolated from the proband (II-2) fibroblast cultures. $(A)$ Pepsin-resistant collagen $\alpha$-chains were separated by $0.5 \mathrm{M}$ urea, $7.5 \%$ SDS-PAGE under unreduced $(-D T T)$, reduced $(+D T T)$, or delayed reduction (delayed $+D T T)$ conditions. The abnormal band, designated $\alpha 1$ (cys) $)_{2}$, migrates between $\alpha 1$ (III) and $\alpha 1(\mathrm{I})$ under unreduced and delayed reduction conditions. The Std is chick type I collagen standard and $C$ is a normal age- and sexmatched control.
Table I. Collagen $\alpha$-Chain Ratios in Dermal Fibroblast Cultures

\begin{tabular}{|c|c|c|c|}
\hline Fibroblast & & $\alpha 1(\mathrm{I}) / \alpha 2(\mathrm{I})$ & $\mathrm{III} / \alpha 1(\mathrm{I})$ \\
\hline Proband (II-2) & & $1.56(0.18)$ & $0.146(0.010)$ \\
\hline Control A & & $2.20(0.17)$ & $0.205(0.100)$ \\
\hline B & & $2.49(0.23)$ & $0.140(0.040)$ \\
\hline \multirow[t]{2}{*}{$\mathrm{C}$} & & $2.43(0.26)$ & $0.071(0.020)$ \\
\hline & $\% \alpha 1$ (cys) & $\alpha 1(\text { cys })_{2}+\alpha 1 / \alpha 2$ & $\mathrm{III} / \alpha 1$ (cys) $)_{2}+\alpha \mathrm{I}$ \\
\hline Proband (II-2) & $23.2 \%(0.71)$ & $2.05(0.22)$ & $0.111(0.010)$ \\
\hline
\end{tabular}

Control fibroblast strains A and B are from age- and sex-matched normal controls. Control A was used for gel analysis. Control $\mathrm{C}$ is from normal foreskin fibroblasts. Standard deviations are listed in parentheses. Gel scan data were normalized to chick type I collagen standard that is run on each gel. Percent $\alpha 1$ (cys) ${ }_{2}$ is calculated as the ratio of the abnormal band to the sum of normal $\alpha 1(\mathrm{I})+\alpha 1(\mathrm{cys})_{2}$.

ing, anomalous band (Fig. 2, $-D T T$ ). This band was observed to merge with the normal $\alpha$-chains upon prereduction of the sample with DTT (Fig. $2,+D T T$ ). It was noted to be independent from $\alpha 1$ (III) when DTT was added after the tracking dye had migrated one-third of the way through the gel (Fig. 2, delayed reduction). Since normal type I collagen $\alpha$-chains do not contain cysteine, these data indicated the presence of a cysteine residue within the triple helical domain of the proband's type I collagen, here referred to as $\alpha 1$ (cys) ${ }_{2}$. An identical electrophoretic pattern, with the same anomalous band, was observed upon repeating these SDS-PAGE analyses with pepsin-purified collagen from dermal fibroblasts isolated from the two affected children (data not shown).

Densitometric analysis of the delayed reduced gel (Fig. 2, delayed $+D T T$ ) demonstrated that a normal ratio of $\alpha 1(\mathrm{I}) /$ $\alpha 2(\mathrm{I})$ and $\alpha 1(\mathrm{III}) / \alpha 1$ (I) was obtained when the scan values for the $\alpha 1$ (cys) $)_{2}$ band were added to the $\alpha 1$ (I) band (Table I). Assuming that intracellular degradation of the mutant $\alpha 1$ (I) was not significant, the theoretical ratio for $\alpha 1$ (I): $\alpha 2(\mathrm{I}): \alpha 1$ (cys) $)_{2}$ as predicted by Steinmann et al. for such a mutation would be 3:2:1 (26). Indeed, the observed ratio for the proband was 1:0.64:0.31, indicating that (a) the $\alpha 1$ (cys) ${ }_{2}$ dimer formation occurred during chain assembly, $(b) \alpha$-chain synthesis in these cells was balanced, and $(c)$ one of the two alleles carried the mutation. Two-dimensional 7.5\% SDS-PAGE with delayed reduction in the first dimension demonstrated that $\alpha 1$ (cys) ${ }_{2}$ comigrated with normal $\alpha(\mathrm{I})$ when fully reduced in the second dimension (data not shown). These data indicated that the $\alpha 1$ (cys) ${ }_{2}$ is consistent with a cysteine containing $\alpha 1$ (I) polypeptide chain.

Incubation of type I collagen $\alpha$-chains with cyanogen bromide selectively cleaves the polypeptides on the $\mathrm{COOH}$ side of methionine residues. This pattern of CB peptides has been well characterized (12). The three species of $\alpha$-chains were separated by delayed reduction 7.5\% SDS-PAGE followed by in situ cleavage with CB. The CB peptides were resolved by 10 $15 \%$ gradient SDS-PAGE without DTT. The CB map obtained from $\alpha 1$ (cys) $)_{2}$ demonstrates a normal pattern of the $\alpha 1$ (I) CB peptides $8,3,7$, and 6 (Fig. 3). However, the presence of higher molecular weight, partially cleaved CB peptides was also ob- 


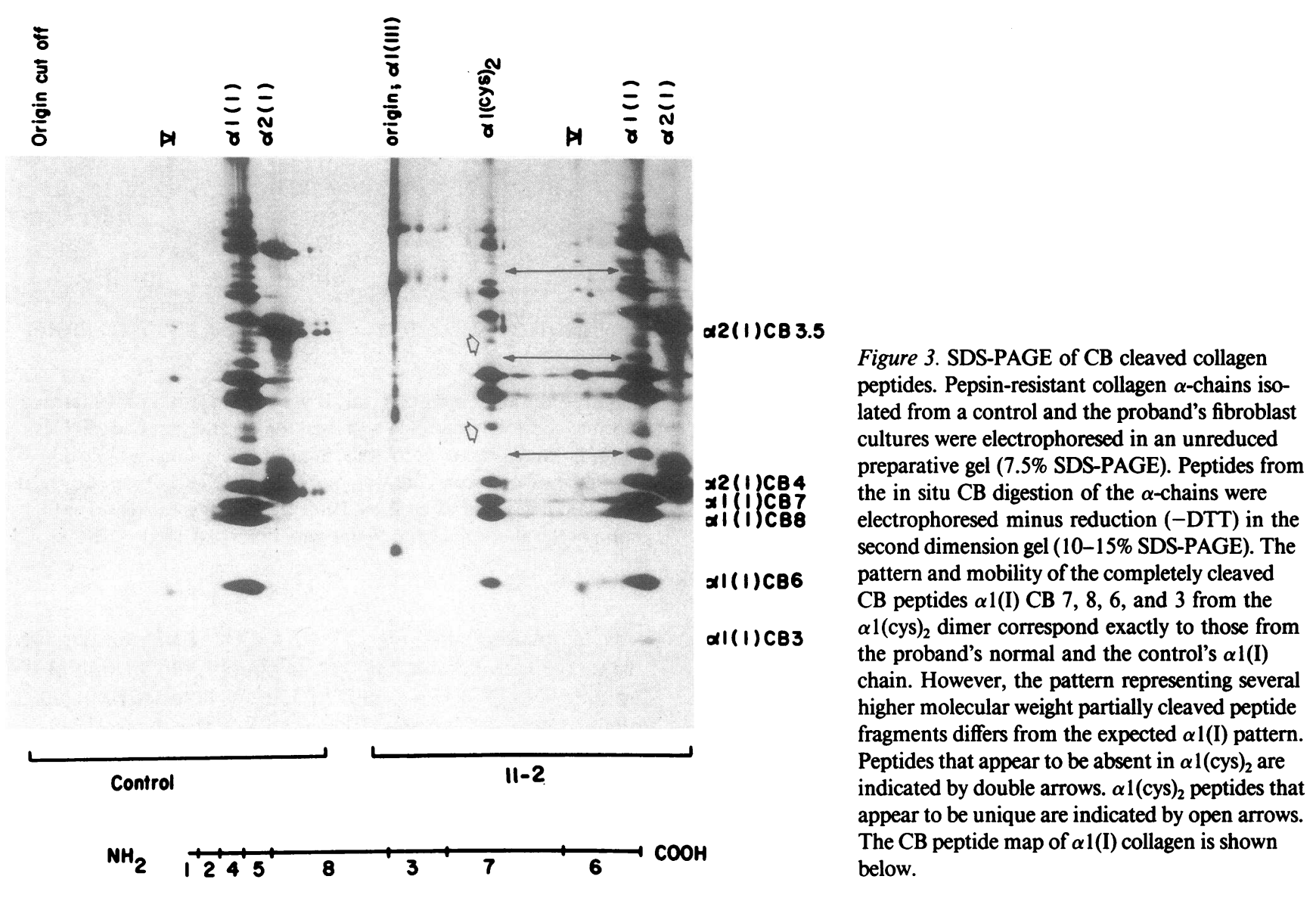

served (see arrows). These data suggested that the cysteine mutation was localized in the smaller fragments derived from the $\mathrm{NH}_{2}$-terminal region of the triple helix (Fig. 3, diagram below figure). These smaller, $\mathrm{NH}_{2}$-terminal $\alpha 1$ (I) CB peptides 1, 2, 4, and 5 are not retained even in 10-15\% gradient SDS-PAGE. However, the partially digested $C B$ peptides would be expected to demonstrate abnormal mobility due to the cysteine dimerization of the partially cleaved $\mathrm{CB}$ fragments.

Molecular analysis. PCR amplification of cDNAs derived from fibroblast mRNA was used to further examine the area of interest in the proband's $\alpha 1$ (I) gene. The $\alpha 1$ (I) helical domain begins at exon 6 . The CB peptides $1,2,4$, and 5 are encoded by exons $6,7-8,9-11$, and 12-13, respectively. We selected oligonucleotides that flanked exons 6-15 in order to amplify a 538bp cDNA that had been reverse transcribed from proband and control fibroblasts. Since the hydroxylamine modification:piperidine cleavage method described by Cotton et al. cleaves mismatched cytosine nucleotides in cDNA heteroduplexes, hybridization of radiolabeled wild-type cDNA probes to the proband's cDNA would be expected to detect a mutated guanosine residue in the coding strand (19). Fig. 4 shows that a 201-bp fragment was observed at reaction times of 30 and $60 \mathrm{~min}$, but was absent when the probe was protected with a cDNA fragment derived from control mRNA. This technique further localized the mutation to $\sim 200$ bp near the $5^{\prime}$ end of the exon 6-15 fragment, and identified it as the substitution of a $G$ with either a $\mathrm{T}$ or an $\mathrm{A}$ residue.

The exon 6-15 cDNA fragment from the proband was cloned by blunt-end ligation into a pBlueScript II KS(+) plas- mid and amplified in a bacterial vector and the plasmid harvested for double-stranded DNA sequencing. Nucleotide sequencing of isolated proband cDNA clones revealed a $\mathrm{G} \rightarrow \mathrm{T}$ mutation in the first position of codon 43. This replaced the expected glycine $43(G G T)$ residue with a cysteine (TGT) (Fig. 5). A total of four clones representing different PCRs were subsequently sequenced. Two of the clones demonstrated the mutation and two had a normal nucleotide sequence, representing the proband's normal and mutant alleles.

Protein structural analysis. The impact of this mutation on the type I collagen triple helix was studied by the determination of thermal stability. The thermal stability of total (normal plus disulfide bonded) collagen triple helix was measured subsequent to isolation by limited pepsin digestion. Fig. 6 demonstrates the thermal stability versus incubation temperature, with these data expressed as a percentage of a sham incubated control. In the control fibroblasts the curve was shifted slightly to the left and $\mathrm{T}_{\mathrm{m}}(50 \%)$ was $40^{\circ} \mathrm{C}$ versus $41.3^{\circ} \mathrm{C}$, indicating a small decrease in thermal stability of the type I collagen triple helix.

\section{Discussion}

During the past 15 years studies from several laboratories (7, 20) have demonstrated multiple mutations affecting the COL1A1 and COL1A2 genes in the setting of osteopenia and skeletal deformity consistent with OI. These mutations presumably affect the proper intracellular and/or extracellular process- 
$\begin{array}{llllll}0 & 30 & 60 & 0 & 30 & 60\end{array}$
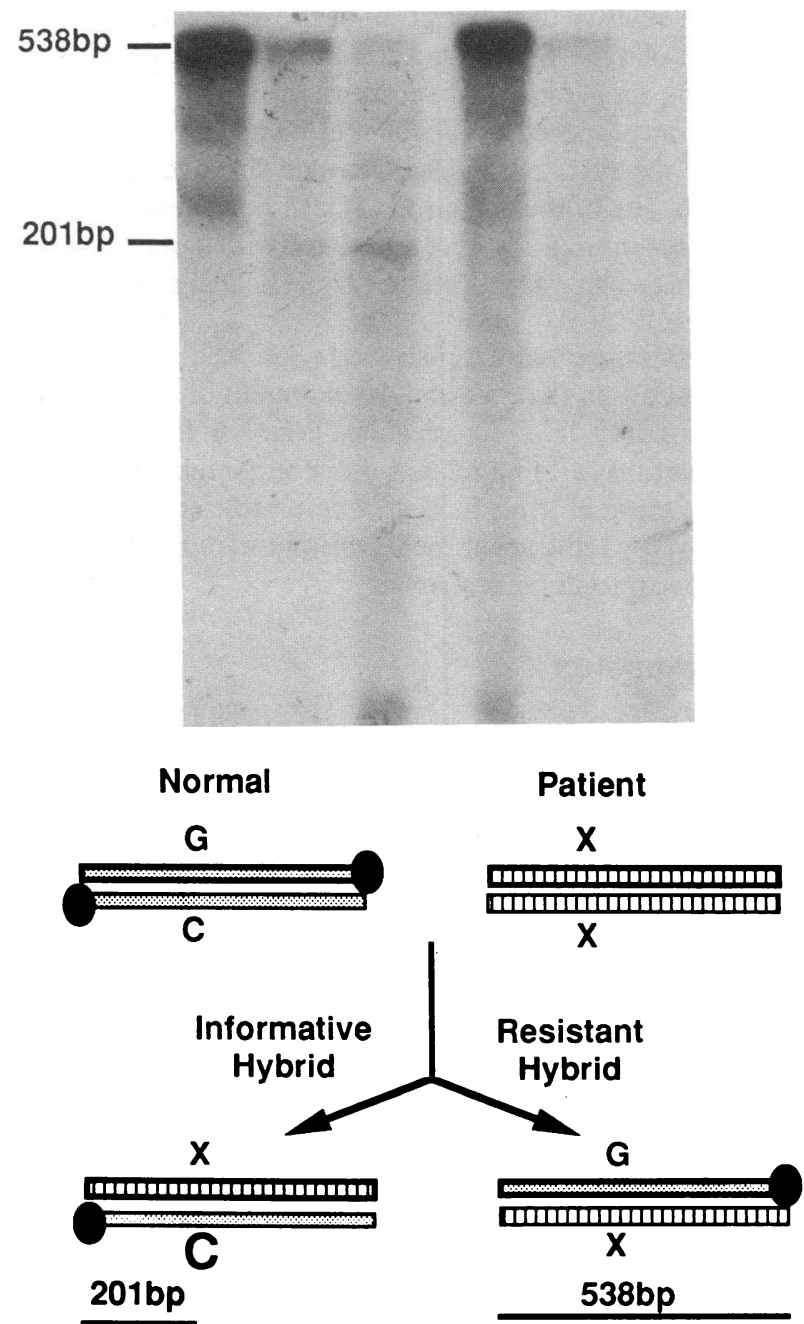

Figure 4. Chemical cleavage of proband cDNA. cDNAs of exon 6-15 from a control and the proband were amplified by PCR. The complimentary wild-type cDNA was labeled with T4 polymerase and used to protect the proband (three left lanes) and control (three right lanes) cDNAs. After 30 and 60 min exposure to hydroxylamine, modification of the probe was revealed by cleavage with piperidine and analysis on 5\% denaturing acrylamide gels. The diagram below indicates that the unpaired $\mathrm{C}$ from the control cDNA is the informative hybrid. $\mathrm{X}$ refers to the sequence in question as unknown. It maps the relative position ( $200 \mathrm{bp}$ into the exon 6-15 fragments) of the mutation within the proband's DNA fragment.

ing of type I collagen and therefore interfere with normal skeletal development and mineralization. In part because of the marked variation in the expression of OI phenotypes, attempts to correlate the size, location, or composition of these mutations with the clinical phenotype have met with little success. Because of considerable overlap in the clinical features of mild OI and idiopathic osteoporosis, the question has surfaced as to whether certain subjects presenting with osteopenia of unknown origin harbor mutations in the type I collagen genes $(2,3)$.

This family is of particular interest because of the extremely mild nature of their skeletal disorder and the fact that their syndrome has features of OI type I: osteopenia, short stature,
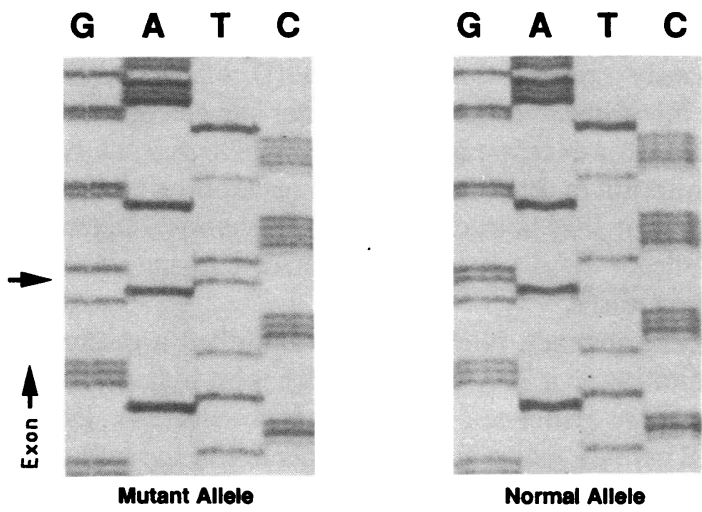

Exon 9 (part|al)

Cys

Normal Allele

GGT CCC ATG GGT CCC CGA GGT CCC CCA GGT CCC CCT GGA AAG Gly Pro Met Gly Pro Arg Gly Pro Hyp Gly Pro Hyp Gly Lys Helical Codon Number 43

Figure 5. DNA sequencing of proband cDNA. Exon 6-15 cDNA from the proband and a control was cloned into a pBlueScript II $\mathrm{KS}(+)$ vector and amplified, and isolated dsDNA was used for sequencing. The arrow on the left of the figure indicates the position of the $\mathrm{G} \rightarrow \mathrm{T}$ that results in glycine (GGT) being mutated to cysteine (TGT). The nucleotide sequence below the figure indicates the position of the mutation to the first position of amino acid 43 (within exon 9). The CB4 peptide begins at the methionine (Met) codon.

blue sclerae, and joint laxity. The absence of fractures in this family is, however, distinctly unusual since most patients with OI experience fractures during the first decade. Patterson has observed that 235 of 249 OI patients of all types had experienced a first fracture before the age of $5 \mathrm{yr}(21)$. Furthermore, it is notable that ascertainment of the 38 -yr-old proband was based on the radiologic evidence of osteoporosis in the absence of complaints referable to the skeletal system. The syndrome of osteoporosis and scoliosis in association with a familial cluster-

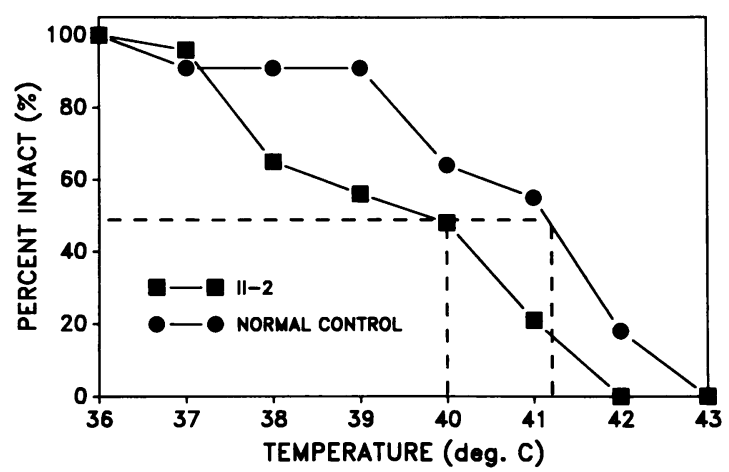

Figure 6. Thermal stability of type I collagen triple helix versus temperature. Pepsinized collagen was incubated at varying temperatures and then subjected to digestion by trypsin:chymotrypsin to probe the stability of the triple helix; in the case of the proband, a mixture of disulfide-linked triple helix and normal triple helical molecules. The undigested protein was electrophoresed and the densitometric area of $\alpha 1(\mathrm{I})+\alpha 2(\mathrm{I})$ was expressed as a percentage of a sham-incubated control. The proband's ( $\square$ ) curve has shifted to the left as compared with the age-matched normal control $(0)$, indicating slightly decreased thermal stability. 
ing of this phenotype has been clinically observed (22). We have previously reported abnormal pro $\alpha 2$ (I) collagen synthesis in a family in which a mother and daughter had osteopenia and scoliosis (23). However, we have been unable to detect a type I collagen mutation in other families with osteoporosis and joint laxity, suggesting that both clinical and biochemical heterogeneity complicate definition of the putative connective tissue lesion in these cases.

In a recent abstract, Nicholls et al. described a glycine to cysteine mutation in a 30-yr-old man with joint hypermobility and radiographic evidence of spinal osteoporosis (24). Type I collagen CB peptide mapping failed to localize to the mutation to any of the major CB peptides. CDNA sequencing of the $\mathrm{NH}_{2}$-terminal region identified a $\mathrm{G} \rightarrow \mathrm{T}$ mutation in glycine residue 19, which corresponds to $\alpha$ (I) CB peptide 2. The triple helical type I collagen was reported to have normal thermal stability which may reflect the extreme $\mathrm{NH}_{2}$-terminal position of that mutation. Thus the mild nature of the patient's disease in their report, along with the location of that mutation within the $\mathrm{NH}_{2}$-terminal region of the collagen triple helix, is similar to the proband described herein.

Spotila et al. have reported a 52-yr-old postmenopausal woman with severe osteopenia, vertebral compression fracture, blue sclerae, slight hearing loss, and a history of five previous fractures beginning at age 7 (25). A serine for glycine substitution in triple helix amino acid position 661 was detected in the $\alpha 2$ (I) chain. While clinically this patient probably represents mild OI type I that presumably escaped diagnosis at a younger age, the interesting feature is that skeletal disease of a mild nature can result from a mutation near the $\mathrm{COOH}$ terminus. In contrast to $\mathrm{NH}_{2}$-terminal mutations, those near the $\mathrm{COOH}$ terminus are considered to cause more disruption of normal triple helix assembly and consequently result in more severe skeletal disease. However, an additional exception is the glycine to cysteine mutation described by Steinmann et al. (26). The mild nature of the disease in their subject was initially considered to be due to a cysteine mutation in the $X$ or $Y$ position of the (Gly-X-Y) $)_{n}$ triplet. This mutation was subsequently localized by Cohn et al. and Labhard et al. to amino acid 1,017, within the $\mathrm{COOH}$ telopeptide region of the $\alpha 1(\mathrm{I})$ chain $(27,28)$.

Cysteine for glycine substitutions in the $\alpha 1$ chain of type I collagen of OI patients have been readily detected on SDSPAGE because dimerization, secondary to disulfide bridge formation, occurs when the heterotrimer contain two mutant chains. This slows the electrophoretic migration of the molecule. Mutations in the $\alpha 2$ (I) chain are less easily detected. First position cysteine mutations in the $\alpha 1$ (I) chain resulting in mild OI have been reported at $\alpha 1$ (I) amino acid 94 (29) and $\alpha 1$ (I) 175 (30). Cysteine mutations have also been found in severe OI type III at $\alpha 1$ (I) 415 (29), 526 (29), and in the lethal OI type II at $\alpha 1$ (I) 988 (27), 748 (31), 904 (32), and 718 (29). In contrast to the mild disease with a $\mathrm{COOH}$-terminal $\alpha 2$ (I) serine for glycine substitution described by Spotila et al. (25) these cases appear to support the postulate of more severe disease with a $\mathrm{COOH}$ terminal mutation.

The impact of cysteine mutations on the assembly of the procollagen triple helix and subsequent development of the extracellular matrix is not well understood. Traub and Steinmann constructed molecular models of mutant $\alpha 1$ (I) chains containing $\mathrm{COOH}$-terminal disulfide bonded cysteine residues (33). These models predict disruption of the molecule in a small region of interchain contacts and adjacent interchain hydrogen bonds relative to the position of the $\alpha 2$ (I) chain. A different model of type I procollagen containing a $\mathrm{COOH}$-terminal cysteine for glycine 748 substitution in a "phase shift" orientation was proposed by Vogel et al. to account for distal distortion of the $\mathrm{NH}_{2}$ proteinase cleavage site over $225 \mathrm{~nm}$ away from the site of the mutation (31). In both instances the $\mathrm{COOH}$-terminal mutations were associated with lethal OI; neither model sheds light on procollagen triple helical structure in milder disease.

The overriding question remains the relationship of the cysteine substitution on the eventual development of osteopenia. Is there a secondary defect in osteoblast function, for example, in the elaboration of bone-related, noncollagenous proteins? Or is the distortion of triple helix structure sufficient to impair the process of type I collagen mineralization and normal organization of the extracellular matrix?

\section{Acknowledgments}

This work was supported in part by National Institutes of Health grants RO1s AR-38370, AR-39870, AR-30426, and AR38933, the Northeast and Washington, DC Area Osteogenesis Imperfecta Foundations, and the Saint Vincent Hospital (Worcester, MA) Research Foundation.

\section{References}

1. Sillence, D. O., A. Senn, and D. M. Danks. 1979. Genetic heterogenity in osteogenesis imperfecta. J. Med. Genet. 16:101-108.

2. Shapiro, J. R., and D. W. Rowe. 1984. Imperfect osteogenesis and osteoporosis. N. Engl. J. Med. 310:1738-1740.

3. Shapiro, J. R., D. W. Rowe, J. Healey, and V. E. Burn. 1987. Familial osteoporosis: pedigrees. J. Bone Miner. Res. 2:S344. (Abstr.)

4. Prockop, D. J. 1988. Osteogenesis imperfecta: a model for genetic causes of osteoporosis and perhaps several other common diseases of connective tissue. Arthritis Rheum. 31:1-8.

5. Smith, R., M. J. O. Francis, and G. R. Houghton. 1983. The Brittle Bone Syndrome. Osteogenesis Imperfecta. Butterworth \& Co., Ltd. London. 44.

6. Byers, P. H., J. F. Bonadio, D. H. Cohn, B. J. Starman, R. J. Wenstrup, and M. C. Willing. 1988. Osteogenesis imperfecta: the molecular basis of clinical heterogeneity. Ann. NY Acad. Sci. 543:117-128.

7. Byers, P. H. 1990. Brittle bones-fragile molecules: disorders of collagen gene structure gene structure and expression. Trends Genet. 6:293-300.

8. Nordin, B. E. 1987. Definition and diagnosis of osteoporosis. Calcif. Tissue Int. 40:57-58.

9. Rubin, K. R., V. M. Schirduan, P. Gendreau, and G. P. Dalsky. 1989. Determinants of bone density in healthy children and adolescence. $J$. Bone Miner. Res. 4:S373. (Abstr.)

10. Shapiro, J. R., V. E. Burn, S. D. Chipman, J. B. Jacobs, B. Schloo, L. Reid, N. Larsen, and F. Louis. 1989. Case report: pulmonary hypoplasia and osteogenesis imperfecta type II with defective synthesis of $\alpha 1$ (I) procollagen. Bone (Elmsford). 10:165-171.

11. Sykes, B. C., B. Puddle, M. J. O. Francis, and R. Smith. 1976. The estimation of two collagens from human dermis by interrupted gel electrophoresis. Biochem. Biophys. Res. Commun. 72:1472-1480.

12. Nikodem, V., and J. R. Fresco. 1979. Protein fingerprinting by SDS-gel electrophoresis after partial fragmentation with CNBr. Anal. Biochem. 97:382386.

13. Laskey, R. A., and A. D. Mills. 1975. Quantitative film detection of ${ }^{3} \mathrm{H}$ and ${ }^{14} \mathrm{C}$ in polyacrylamide gels by fluorography. Eur. J. Biochem. 56:335-341.

14. Bruckner, P., and D. J. Prockop. 1981. Proteolytic enzymes as probes for the triple-helical conformation of procollagen. Biochemistry. 110:360-368.

15. Rowe, D. W., and B. E. Kream. 1982. Regulation of collagen synthesis in fetal rat calvaria by 1,25-dihydroxyvitamin $\mathrm{D}_{3}$. J. Biol. Chem. 257:8009-8015.

16. D'Alessio, J. M., M. C. Noon, H. L. Ley, and G. F. Gerard. 1987. Comparison of cDNA synthesis by avian and cloned murine reverse transcriptase. Focus (Idaho). 9:1-4.

17. Scharf, S. J., G. T. Hern, and H. A. Erlich. 1986. Direct cloning and sequence analysis of enzymatically amplified genomic sequences. Science (Wash. DC). 233:1076-1078.

18. Maniatis, T., E. Fritsch, and J. Sambrook. 1982. Molecular Cloning: A Laboratory Manual. Cold Spring Harbor Laboratory, Cold Spring Harbor, NY. 545 pp. 
19. Cotton, R. G., N. R. Rodrigues, and R. D. Campbell. 1988. Reactivity of cytosine and thymine in a single base pair mis-matches with hydroxylamine and osmium tetroxide and its application to the study of mutations. Proc. Natl. Acad. Sci. USA. 85:4397-4401.

20. Kuivaniemi, H., G. Tromp, and D. J. Prockop. 1991. Mutations in collagen genes: causes of rare and some common diseases in humans. FASEB (Fed. Am. Soc. Exp. Biol.) J. 5:2052-2060.

21. Patterson, C. R. 1978. Osteogenesis imperfecta and fractures in childhood. Health Visitor. 51:174-176.

22. Velis, K. P., J. H. Healey, and R. Schneider. 1989. Peak skeletal mass assessment in young adults with idiopathic scoliosis. Spine. 14:706-711.

23. Shapiro, J. R., V. E. Burn, S. D. Chipman, K. P. Velis, and M. Bansal. 1989. Osteoporosis and familial idiopathic scoliosis: association with an abnormal $\alpha 2$ (I) collagen. Connect. Tissue Res. 21:117-124.

24. Nicholls, A. C., J. Oliver, D. Renouf, and F. M. Pope. 1990. Type I collagen mutations in osteogenesis imperfecta and inherited osteoporosis. Vth Int. Conf. Osteogensis Imperfecta. Pavia, Italy. (Abstr.).

25. Spotila, L. D., C. D. Constantinou, L. Serede, A. Ganguly, B. D. Riggs, and D. J. Prockop. 1991. Mutation in a gene for type I procollagen (COL1A2) in a woman with postmenopausal osteoporosis: evidence for phenotypic and genotypic overlap with mild osteogenesis imperfecta. Proc. Natl. Acad. Sci. USA. 88:5423-5427.

26. Steinmann, B., A. Nicholls, and F. M. Pope. 1986. Clinical variability of osteogenesis imperfecta reflecting molecular heterogeneity: cysteine substitutions in the alpha 1(I) collagen chain producing lethal and mild forms. J. Biol. Chem. 261:8958-8964.

27. Cohn, D. H., S. Apone, D. R. Eyre, B. J. Starman, P. Andreassen, H. Charbonneau, A. C. Nicholls, F. M. Pope, and P. H. Byers. 1988. Substitution of cysteine for glycine within the carboxyl-terminal telopeptide of the alpha 1 chain of type I collagen produces mild osteogenesis imperfecta. J. Biol. Chem. 263:14605-14607

28. Labhard, M. E., M. K. Wirtz, F. M. Pope, A. C. Nicholls, and D. W. Hollister. 1988. A patient with mild dominantly inherited osteogenesis imperfecta. Mol. Biol. Med. 5:197-207.

29. Starman, B. J., D. Eyre, H. Charbonneau, M. Harrylock, M. A. Weis, L. Weiss, J. M. Graham, and P. H. Byers. 1989. Osteogenesis imperfecta. The position of substitution for glycine by cysteine in the triple helical domain of the pro $\alpha$ (I) chains of type I collagen determines the clinical phenotype. J. Clin. Invest. 84:1206-0000.

30. deVries, W. N., and W. J. deWet. 1986. The molecular defect in an autosomal dominant form of osteogenesis imperfecta: synthesis of type I procollagen containing cysteine in the triple helical domain of proal(I) chains. J. Biol. Chem. 261:9056-9064.

31. Vogel, B. E., R. Doelz, K. E. Kadler, Y. Hojima, J. Engel, and D. J. Prockop. 1987. A substitution of cysteine for glycine 748 of the $\alpha 1$ chain produces a kink at this site in the procollagen I molecule and an altered $\mathrm{N}$-proteinase cleavage site over $225 \mathrm{~nm}$ away. J. Biol. Chem. 263:19249-19255.

32. Constantinou, C. D., K. B. Nielsen, and D. J. Prockop. 1989. A lethal variant of osteogenesis imperfecta has a single base mutation that substitutes cysteine for glycine 904 of the $\alpha 1$ (I) chain of type I procollagen. The asymptomatic mother has an unidentified mutation producing an overmodified and unstable type I procollagen. J. Clin. Invest. 83:574-584.

33. Traub, W., and B. Steinmann. 1986. Structural study of a mutant type I collagen from a patient with lethal osteogenesis imperfecta containing an intramolecular disulfide bond in the triple helical domain. FEBS (Fed. Eur. Biochem. Soc.) Lett. 198:213-216. 